\title{
Preparation and Properties of Cross-Linked Starch Nanocrystals/Polylactic Acid Nanocomposites
}

\author{
Zuodong Yin, ${ }^{1}$ Jiliang Zeng, ${ }^{1,2}$ Cai Wang, ${ }^{2}$ and Zelin Pan ${ }^{2}$ \\ ${ }^{1}$ School of Chemistry and Chemical Engineering, Guangxi University, Nanning 530004, China \\ ${ }^{2}$ Beijing National Laboratory for Molecular Sciences (BNLMS), Key Laboratory of Green Printing, Institute of Chemistry, \\ Chinese Academy of Sciences, Beijing 100190, China
}

Correspondence should be addressed to Zuodong Yin; zdyin_gu@163.com and Cai Wang; wangc@iccas.ac.cn

Received 19 January 2015; Accepted 19 March 2015

Academic Editor: Jiguang Liu

Copyright (c) 2015 Zuodong Yin et al. This is an open access article distributed under the Creative Commons Attribution License, which permits unrestricted use, distribution, and reproduction in any medium, provided the original work is properly cited.

\begin{abstract}
Cross-linked starch nanocrystals (CStN)/polylactic acid (PLA) nanocomposites were prepared by blending CStN and PLA, wherein CStN were homogeneously dispersed in the nanocomposites. The structure and morphology of the nanocomposites were studied through FTIR, XRD, and SEM. The results of mechanical test showed that the strength and toughness of the nanocomposites could be improved when the additive amount of hydrophobic CStN was 3\% (weight ratio). Meanwhile, the microstructures of tensile fracture surface showed that CStN were uniformly dispersed in PLA matrix, and the tensile fracture surface was presented as ductile fracture. Moreover, water vapor permeability experiments illustrated that the addition of CStN reduced the water vapor permeability of PLA nanocomposites, so CStN have some resistance to water vapor. Those above indicated that CStN have functions of reinforcement and toughness in PLA matrix; therefore, they are expected to be used as functional additives in polymer matrix such as PLA, polycaprolactone (PCL), and polybutylene succinate (PBS).
\end{abstract}

\section{Introduction}

As a kind of natural polymer, starch is of low price, biodegradable, and rich of sources and also has a broad industrial application. To study the preparation and application of functional starch derivatives, especially using industrial starch as raw material, will have a more extensive application prospect. Starch nanocrystals [1-3] are a kind of natural starch derivative and have a high crystallinity and hydrophilic structure of platelet, and they attract much attention of many researchers. Researchers have proceeded chemical modifications on the surface of starch nanocrystals and have prepared fatty acid modified starch nanocrystals [4], acetylated starch nanocrystals [5], polycaprolactone grafted starch nanocrystals $[6,7]$, polylactic acid grafted starch nanocrystals [8], polystyrene grafted starch nanocrystals $[9,10]$, cross-linked starch nanocrystals [11], and so on to improve the compatibility and the dispersion stability of starch nanocrystals in polymer matrix. It shows that the mechanical properties of the nanocomposites obtained by blending the prepared polycaprolactone grafted starch nanocrystals with polylactic acid (PLA) are enhanced obviously in literature [7].

In this paper, hydrophobic cross-linked starch nanocrystals were prepared through a simple cross-linking modification reaction, and, then, they were blended with PLA to get CStN/PLA nanocomposites. The structure, mechanical properties, and micromorphology of the nanocomposites were studied, and the effects of CStN on the nanocomposites were also analyzed; moreover, the effect of CStN on water vapor permeability of the nanocomposites was researched by cup method.

\section{Materials and Methods}

The corn starch with the moisture content of $14 \%$ was industrial products and purchased from Beijing Hongxing Starch Factory. Epichlorohydrin was supplied by Beijing Chemical Works and its grade was AR. The polylactic acid (PLA), whose thermal deformation temperature was $56^{\circ} \mathrm{C}$, was sheet and from Shenzhen Guanghua Weiye Industrial 
Co., Ltd. (China). Other reagents were all commercially available chemical reagents.

2.1. Preparation of Cross-Linked Starch Nanocrystals. $180 \mathrm{~g}$ of starch (dry weight) was mixed with $1250 \mathrm{~mL}$ of sulfuric acid $(2.87 \mathrm{M})$ [1]. The suspension was kept with the constant stirring speed under $45^{\circ} \mathrm{C}$ for acid hydrolysis for 7 days. Then the resulting suspension was washed with deionized water by successive centrifugations until the $\mathrm{pH}$ of the suspension was neutral. After the treatment of ultrasonic processor (Ultrasonic Cell Crusher, Type JY92-II, Ningbo XinYi Ultrasonic Equipment Co., Ltd. (China); the maximum power is $650 \mathrm{w}$ ) for $3 \mathrm{~min}$ to ensure that the starch nanocrystals dispersed homogeneously, the aqueous suspension of starch nanocrystals with the weight concentration of about $0.8 \%$ was obtained.

$2500 \mathrm{~mL}$ of the above aqueous suspension (equivalent to $20.0 \mathrm{~g}$ of dry starch) was put into the $5 \mathrm{~L}$ three-neck flask equipped with a reflux condensing tube, and, then, under stirring, the aqueous solution of $\mathrm{NaOH}(5 \%)$ was added to keep the $\mathrm{pH}$ around 10 to 11 . When the temperature of the solution was heated to $30-35^{\circ} \mathrm{C}, 2.0 \mathrm{~g}$ of epichlorohydrin was added. After 2 hours, we measured the $\mathrm{pH}$ and refilled the alkali to keep the $\mathrm{pH}$ around 10-11. Then, hydrochloric acid was added to neutralize after reacting for another 6 hours, and the product was separated by centrifugation and washed with deionized water, acetone, and chloroform, respectively, 3 times for each. Finally, the resulting chloroform suspension of $\mathrm{CStN}$ was obtained, whose concentration was about $0.5 \%$ (wt). The molar ratio of epichlorohydrin (ECH) and starch glucose unit (AGU) was about 0.08 , which was calculated through utilizing the weight changes of starch nanocrystals before and after reaction.

2.2. Preparation of Cross-Linked Starch Nanocrystals/PLA Nanocomposites. Under the stirring, the above chloroform suspensions of CStN with dry weight $0.1 \mathrm{~g}, 0.3 \mathrm{~g}, 0.5 \mathrm{~g}$, and $0.8 \mathrm{~g}$ were separately added to $80 \mathrm{~mL}$ chloroform solution which contained $10 \mathrm{~g}$ PLA, and the chloroform was recycled by gradual distillation. Then, the resulting products were dried in a vacuum at $60^{\circ} \mathrm{C}$ for $4 \mathrm{~h}$, and different additive amounts of CStN/PLA nanocomposites were obtained. Subsequently, they were put on the hydraulic press (doublespeed manual hydraulic press, Type YYS-1, Guoying FengHua Machinery Factory), and the films with the thickness of $0.1-0.3 \mathrm{~mm}$ were molded by hot pressing at $180-190^{\circ} \mathrm{C}$ and marked, respectively, as CStN-PLA-1, CStN-PLA-2, CStNPLA-3, and CStN-PLA-4, whose additive amount of CStN corresponded to $1 \%, 3 \%, 5 \%$, and $8 \%$, respectively.

2.3. Materials Characterization. Fourier transform infrared (FTIR) spectra of the nanocomposites films in the range of $4000-500 \mathrm{~cm}^{-1}$ were recorded on FTIR TENSOR 27 spectra (BRUKER, Germany) with the method of ATR-FTIR.

The XRD patterns of CStN/PLA nanocomposites films were obtained on Rigaku D/max-2500 X-ray diffractometer (Rigaku Corporation, Tokyo, Japan) with $\mathrm{Cu}-\mathrm{K} \alpha$ radiation at
$40 \mathrm{kV}$ and $200 \mathrm{~mA}$. The diffraction data were recorded over the $2 \theta$ range of $2-40^{\circ}$.

The morphologies of fractured surfaces of CStN/PLA nanocomposites after the tensile tests were observed by field emission scanning electron microscopy (FE-SEM, JSM$6701 F$, JEOL, Japan) with the highest resolution of $1 \mathrm{~nm}$. The sample surface was first coated with a thin layer of gold.

According to China National Standards GB/T 1040-2006, the mechanical properties (including tensile modulus, tensile strength, and breaking elongation) of the nanocomposites films were tested on universal tensile tester (Instron 3365 machine, Instron Company, UK) at $50 \mathrm{~mm} \cdot \mathrm{min}^{-1}$. The samples were with the size of $150 \mathrm{~mm} \times 10 \mathrm{~mm}$. At least five specimens were tested for an average value.

Thermal analysis of CStN/PLA nanocomposites was carried out using a DSC Q200 differential calorimeter (TA instruments, USA) equipped with a manual liquid nitrogen cooling system between 0 and $200^{\circ} \mathrm{C}$ at a rate of $20^{\circ} \mathrm{C} \cdot \mathrm{min}^{-1}$. The using dosage of the samples was $7-10 \mathrm{mg}$ and the flow rate of nitrogen was $50 \mathrm{~mL} \cdot \mathrm{min}^{-1}$.

2.4. Water Vapor Permeability (WVP) Test. Water vapor permeability of the nanocomposites films was determined by the method according to China National Standards GB 103788 and the thickness of the films was measured on the basis of China National Standards GB/T6672-2001. The samples were placed in the permeability cup filled with the desiccant $\left(\mathrm{CaCl}_{2}\right)$ and then sealed up and put into a temperature humidity chamber with the temperature of $38 \pm 0.6^{\circ} \mathrm{C}$ and the relative humidity of $90 \pm 2 \%$. There was no need to weigh the samples at intervals until the contiguous mass difference was less than $5 \%$. In accordance with the resulting data, water vapor permeability $(\mathrm{WVP})\left[(\mathrm{g} \cdot \mathrm{m}) /\left(\mathrm{m}^{2} \cdot \mathrm{s} \cdot \mathrm{Pa}\right)\right]$ was calculated by the following formula:

$$
\mathrm{WVP}=\frac{\Delta m \cdot d}{A \cdot t \cdot \Delta p}
$$

where $\Delta m$ (g) was taken for the contiguous mass difference, $d(\mathrm{~m})$ the thickness of the films, $A\left(\mathrm{~m}^{2}\right)$ the valid water vapor permeability area of the films with the value of $0.002826 \mathrm{~m}^{2}$, $t$ (s) the interval time, and $\Delta p(\mathrm{~Pa})$ the pressure difference, whose value was $5966.82 \mathrm{~Pa}$.

\section{Results and Discussion}

3.1. FTIR Spectrum Analysis. The FTIR spectra of CStN/PLA nanocomposites are shown in Figure 1. At $1750 \mathrm{~cm}^{-1}$, all the samples have strong stretching vibration absorption of ester carbonyl in PLA and no obvious difference, which indicates that CStN almost have no effect on the molecule structure of PLA. As compared with PLA, CStN/PLA nanocomposites have slight difference in absorption peaks of $3392 \mathrm{~cm}^{-1}$ (the stretching vibration absorption of $\mathrm{OH}$ in $\mathrm{CStN}$ ) and $1645 \mathrm{~cm}^{-1}$ (the stretching vibration absorption of ether bond in CStN). The absorption peaks of $3392 \mathrm{~cm}^{-1}$ and $1645 \mathrm{~cm}^{-1}$ become more obvious with the increase of additive CStN, which explains that CStN are of good dispersibility in PLA. 


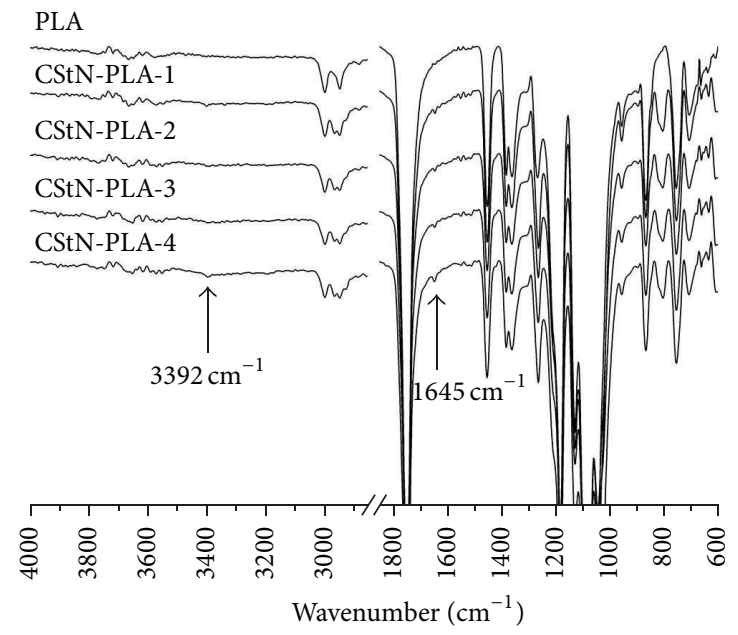

Figure 1: ATR-FTIR spectra of CStN/PLA nanocomposites and PLA films.

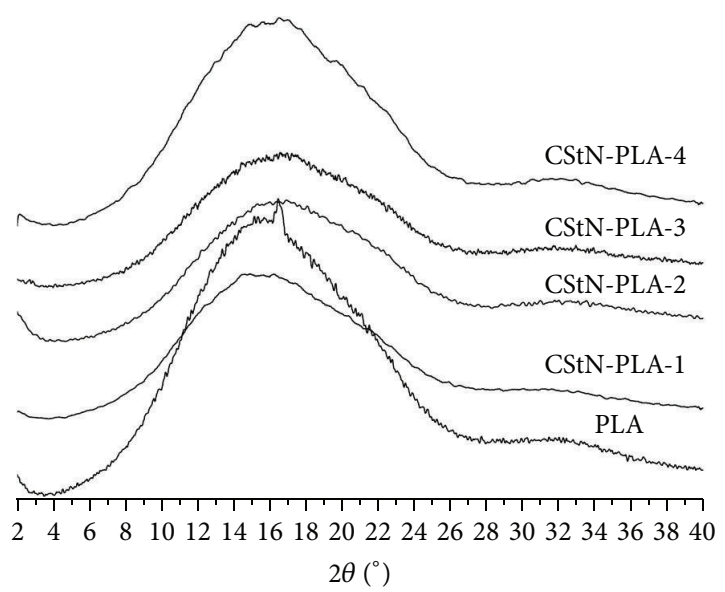

FIGURE 2: X-ray diffraction patterns of CStN/PLA nanocomposites and PLA films.

3.2. WAXD Analysis. X-ray patterns of different compositions are displayed in Figure 2 and there appears no obvious crystal diffraction peak. At $2 \theta$ of $15^{\circ}, 17^{\circ}, 18^{\circ}$, and $21^{\circ}$, there are weak peaks in the diffractogram of CStN-PLA-4, which are the typical diffraction peaks of CStN and further illustrate that the high crystallinity structure of CStN did not alter under the condition of hot pressing process and that the interaction between CStN and PLA matrix is not apparent.

3.3. Morphology of the Nanocomposites Films. The SEM images of fractured morphologies of CStN/PLA nanocomposites are shown in Figure 3. In Figure 3(a), the fractured morphology is clear and relatively smooth, which indicates that CStN hardly influence the interface of nanocomposites and do not play a significant reinforcement. There are many evident uniform microhumps resulting from the ductile fracture found in Figure 3(b), assuming that CStN are compatible with PLA matrix and form a certain interaction that would reinforce and toughen PLA matrix, and it also speculates that the mechanical properties of CStN-PLA2 are superior to those of CStN-PLA-1. It is shown in Figure 3(c) that the fractured morphology is relatively clear and CStN are significantly accumulated and have a relatively poor uniformity in PLA, resulting in the poor mechanical properties of the nanocomposites. In Figure 3(d), there are a lot of obvious uniform microhumps forming from the ductile fracture on the fractured surface, which postulates that there exists a certain interaction between CStN and PLA matrix; nevertheless, the mechanical properties are worse because of the obvious cracks and the accumulation of particles.

\subsection{Mechanical Properties of CStN/PLA Nanocomposites.}

Figure 4 shows the change of the mechanical properties of CStN/PLA nanocomposites. When the additive amount of $\mathrm{CStN}$ is less than $3 \%$, tensile strength, tensile modulus, and breaking elongation are all increased with the rising additive amount. When arriving 3\%, tensile strength of CStN/PLA nanocomposites is increased about $12 \%$, tensile modulus about $35 \%$, and breaking elongation 16\%; namely, the strength and toughness of CStN/PLA nanocomposites are enhanced, which agrees with the microstructure of the nanocomposites that Figure 3 reflects. This is because CStN are compatible and dispersible well in PLA matrix and the interaction force between CStN and PLA is enlarged; therefore, CStN have functions of enhancement and toughness; when the amount is more than $3 \%$, the functions of enhancement and toughness are weakened. The reason is that the dispersity of abundant CStN in PLA is turned worse and CStN begin aggregating, which weakens the interaction force between CStN and PLA. As the amount reaches $8 \%$, the volume fraction of CStN in PLA matrix is increased and the matrix layer of adjacent CStN particles becomes thinner, which makes the interaction force between the particles strengthen, resulting in a high stress triaxiality surrounding the particle and making the shift of molecular chain difficult; sequentially, the tensile modulus of CStN/PLA nanocomposites is larger than that of pure PLA.

3.5. Thermal Performance Analysis. The thermal performances of CStN/PLA nanocomposites are exhibited in Figure 5. The softening temperature of PLA is about $62^{\circ} \mathrm{C}$, and the softening temperature of CStN/PLA nanocomposites is lower, about $53^{\circ} \mathrm{C}$. The melting temperature decreases slightly with the increasing additive amount of CStN. The reason of softening temperature decreased should be mainly because the lubricating effect of nanoscale CStN between PLA molecules reduces the interactions of PLA molecules. CStN do not interact with PLA matrix during the melting process, and the lubricating effect is no more important than the thermal motion of PLA macromolecules, so the increasing additive amount of CStN has little effect on the melting temperature of PLA.

3.6. Water Vapor Permeability Analysis. Table 1 shows the water vapor permeability (WVP) of PLA and different CStN/ PLA nanocomposites films. After blending PLA and CStN, the WVP is all reduced; the reason is that CStN are of 
TABLE 1: Water vapor permeability of CStN/PLA nanocomposites and PLA films.

\begin{tabular}{lccccc}
\hline & CStN/\% & $\Delta m / g$ & $d / \mathrm{m}$ & $t / \mathrm{h}$ & $\mathrm{WVP} /\left(\mathrm{g} \cdot \mathrm{m} /\left(\mathrm{m}^{2} \cdot \mathrm{s} \cdot \mathrm{Pa}\right)\right)$ \\
\hline PLA & 0 & 0.3127 & $1.46 \times 10^{-4}$ & 72 & $10.44 \times 10^{-16}$ \\
CStN-PLA-1 & 1 & 0.0810 & $3.00 \times 10^{-4}$ & 48 & $8.32 \times 10^{-16}$ \\
CStN-PLA-2 & 3 & 0.1789 & $1.51 \times 10^{-4}$ & 48 & $8.23 \times 10^{-16}$ \\
CStN-PLA-3 & 5 & 0.1603 & $1.60 \times 10^{-4}$ & 48 & $8.81 \times 10^{-16}$ \\
CStN-PLA-4 & 8 & 0.1136 & $2.44 \times 10^{-4}$ & 48 & $9.51 \times 10^{-16}$ \\
\hline
\end{tabular}

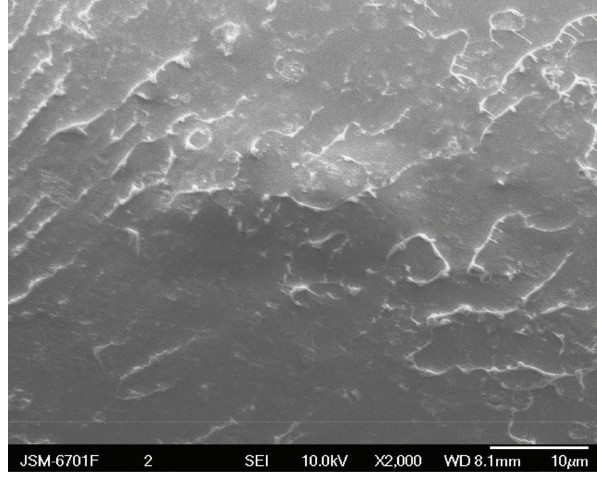

(a) CStN-PLA-1

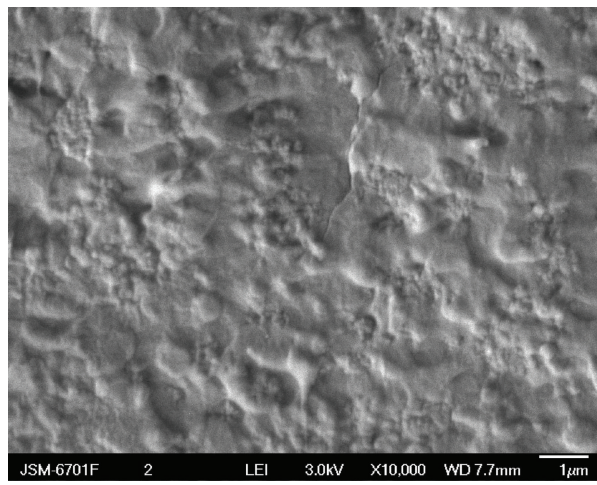

(c) CStN-PLA-3

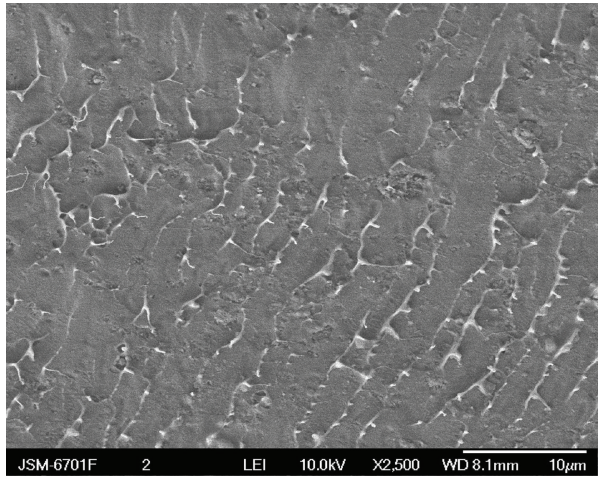

(b) CStN-PLA-2

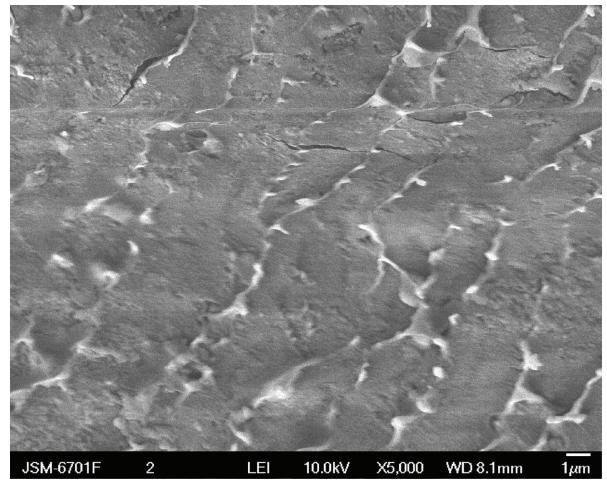

(d) CStN-PLA-4

Figure 3: Morphologies of fractured surfaces of CStN/PLA nanocomposites after the tensile tests.

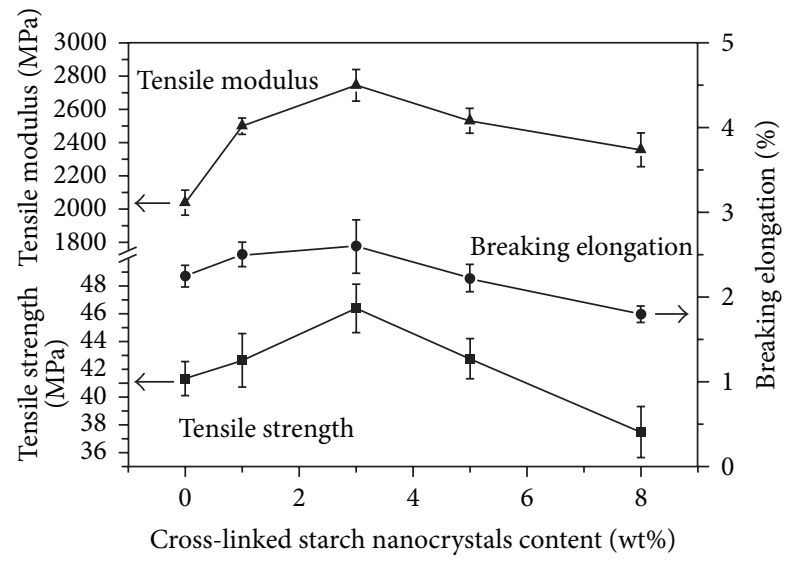

FIGURE 4: Mechanical properties of CStN/PLA nanocomposites.

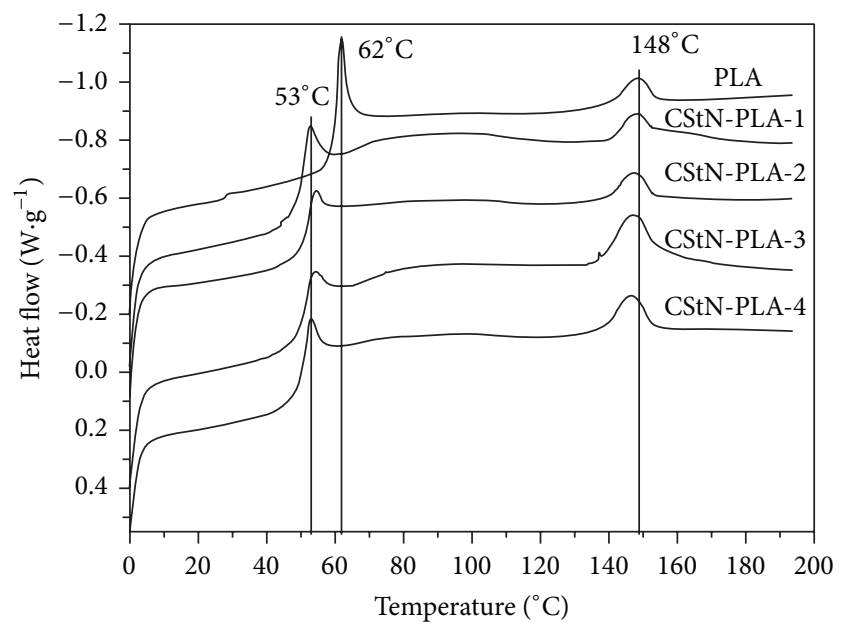

FIGURE 5: DSC thermograms of CStN/PLA nanocomposites and PLA films. 
good hydrophilicity that strengthen the interaction force with water molecule and possess a high crystallinity, which make water molecule difficult to penetrate the sheet of CStN and need to move along the surface of CStN. The uniformly dispersive CStN slow the penetrating speed of water molecule; thus the WVP is decreased. Moreover, water molecules could cluster through personal hydrogen bond [12], which makes water clusters have a larger average size, and then the WVP of PLA nanocomposites is further reduced.

\section{Conclusions}

In cross-linked starch nanocrystals (CStN)/polylactic acid (PLA) nanocomposites, the addition of CStN can significantly improve the dispersive stability and interface compatibility in polymer matrix. When the additive amount of CStN was 3\% (wt), the strength and toughness of PLA nanocomposites were increased. The addition of CStN can significantly reduce the softening temperature of PLA nanocomposites but did have little influence on its melting temperature. The water vapor permeability of CStN/PLA nanocomposites can reduce significantly. As a conclusion, the CStN were expected to be used as a functional biopolymer additive in polymer composites.

\section{Conflict of Interests}

The authors declare that there is no conflict of interests regarding the publication of this paper.

\section{Acknowledgment}

This work was financially supported by the National Nature Science Foundation of China (50573087, 51073167).

\section{References}

[1] H. Angellier, L. Choisnard, S. Molina-Boisseau, P. Ozil, and A. Dufresne, "Optimization of the preparation of aqueous suspensions of waxy maize starch nanocrystals using a response surface methodology," Biomacromolecules, vol. 5, no. 4, pp. 1545-1551, 2004.

[2] H. Angellier-Coussy, J.-L. Putaux, S. Molina-Boisseau, A. Dufresne, E. Bertoft, and S. Perez, "The molecular structure of waxy maize starch nanocrystals," Carbohydrate Research, vol. 344, no. 12, pp. 1558-1566, 2009.

[3] D. Lecorre, J. Bras, and A. Dufresne, "Ceramic membrane filtration for isolating starch nanocrystals," Carbohydrate Polymers, vol. 86, no. 4, pp. 1565-1572, 2011.

[4] H. Namazi and A. Dadkhah, "Convenient method for preparation of hydrophobically modified starch nanocrystals with using fatty acids," Carbohydrate Polymers, vol. 79, no. 3, pp. 731737, 2010.

[5] Y. Xu, W. Ding, J. Liu et al., "Preparation and characterization of organic-soluble acetylated starch nanocrystals," Carbohydrate Polymers, vol. 80, no. 4, pp. 1078-1084, 2010.

[6] H. Namazi and A. Dadkhah, "Surface modification of starch nanocrystals through ring-opening polymerization of $\varepsilon$-caprolactone and investigation of their microstructures," Journal of Applied Polymer Science, vol. 110, no. 4, pp. 2405-2412, 2008.
[7] J. Yu, F. Ai, A. Dufresne, S. Gao, J. Huang, and P. R. Chang, "Structure and mechanical properties of poly(lactic acid) filled with (starch nanocrystal)-graft-poly( $\varepsilon$-caprolactone)," Macromolecular Materials and Engineering, vol. 293, no. 9, pp. 763770, 2008.

[8] N. L. García, M. Lamanna, N. D’Accorso, A. Dufresne, M. Aranguren, and S. Goyanes, "Biodegradable materials from grafting of modified PLA onto starch nanocrystals," Polymer Degradation and Stability, vol. 97, no. 10, pp. 2021-2026, 2012.

[9] S. Song, C. Wang, Z. Pan, and X. Wang, "Preparation and characterization of amphiphilic starch nanocrystals," Journal of Applied Polymer Science, vol. 107, no. 1, pp. 418-422, 2008.

[10] S. W. Song, C. Wang, D. M. Xu et al., "Preparation of polystyrene-g-starch nanocrystals," Chemistry, vol. 72, no. 2, pp. 176179, 2009.

[11] L. Ren, M. Jiang, L. Wang, J. Zhou, and J. Tong, "A method for improving dispersion of starch nanocrystals in water through crosslinking modification with sodium hexametaphosphate," Carbohydrate Polymers, vol. 87, no. 2, pp. 1874-1876, 2012.

[12] Y. L. Wu, G. M. Li, J. F. Li et al., "Transfer behavior of water vapor in polymer membranes and dehumidification of gases by membrane separation," Membrane Science and Technology, vol. 27, no. 3, pp. 1-5, 2007. 

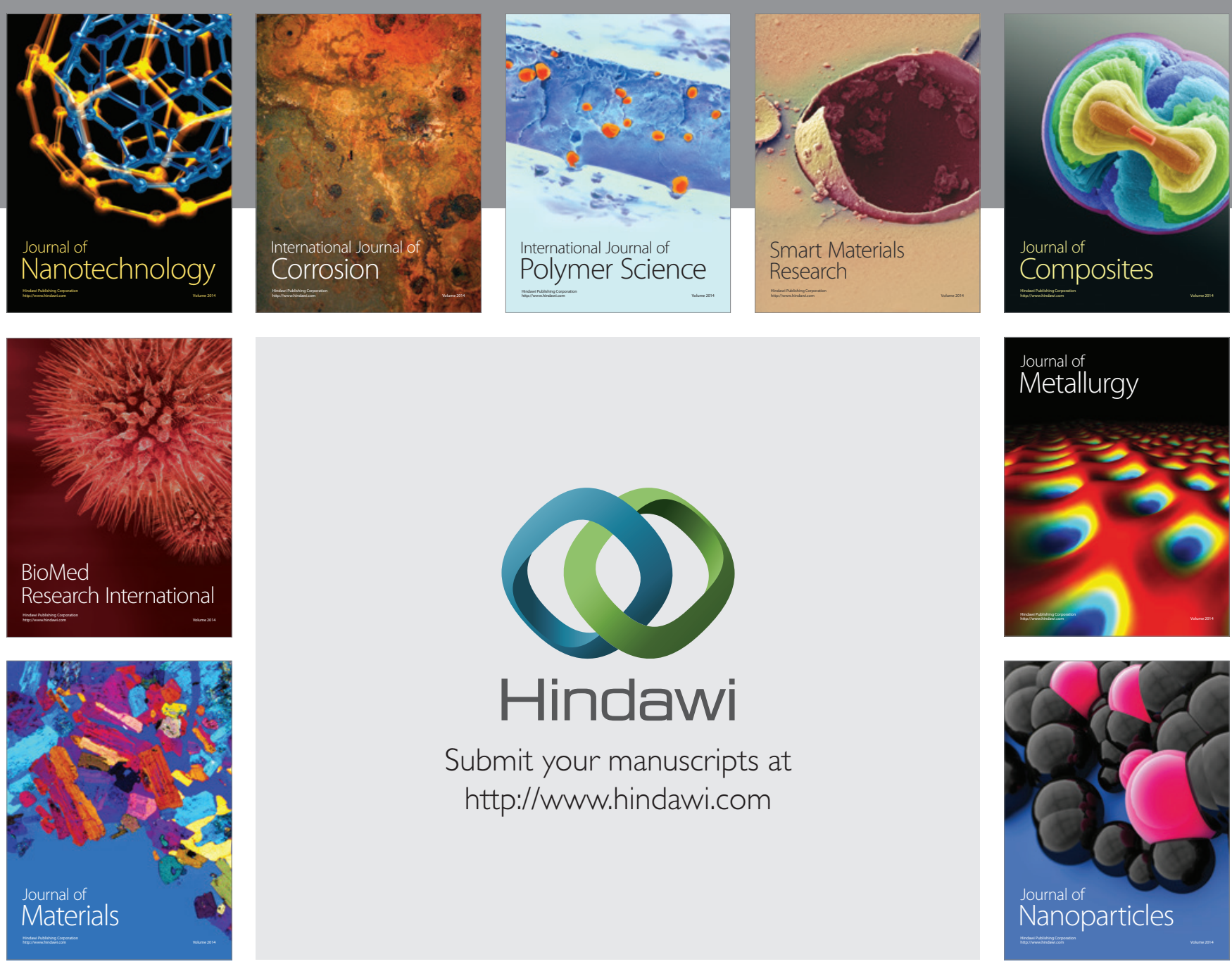

Submit your manuscripts at http://www.hindawi.com
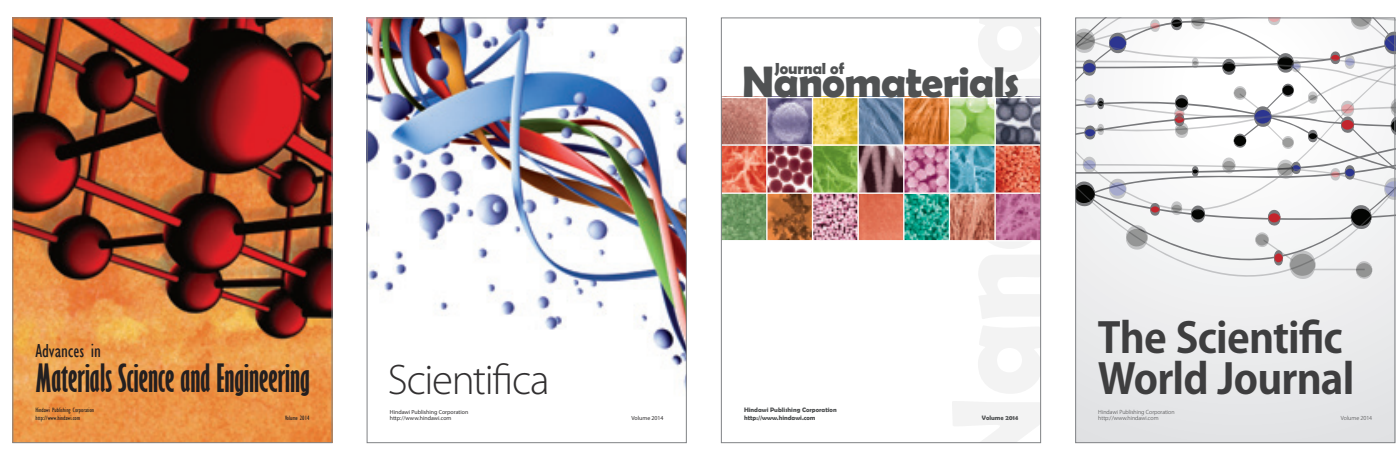

\section{The Scientific World Journal}
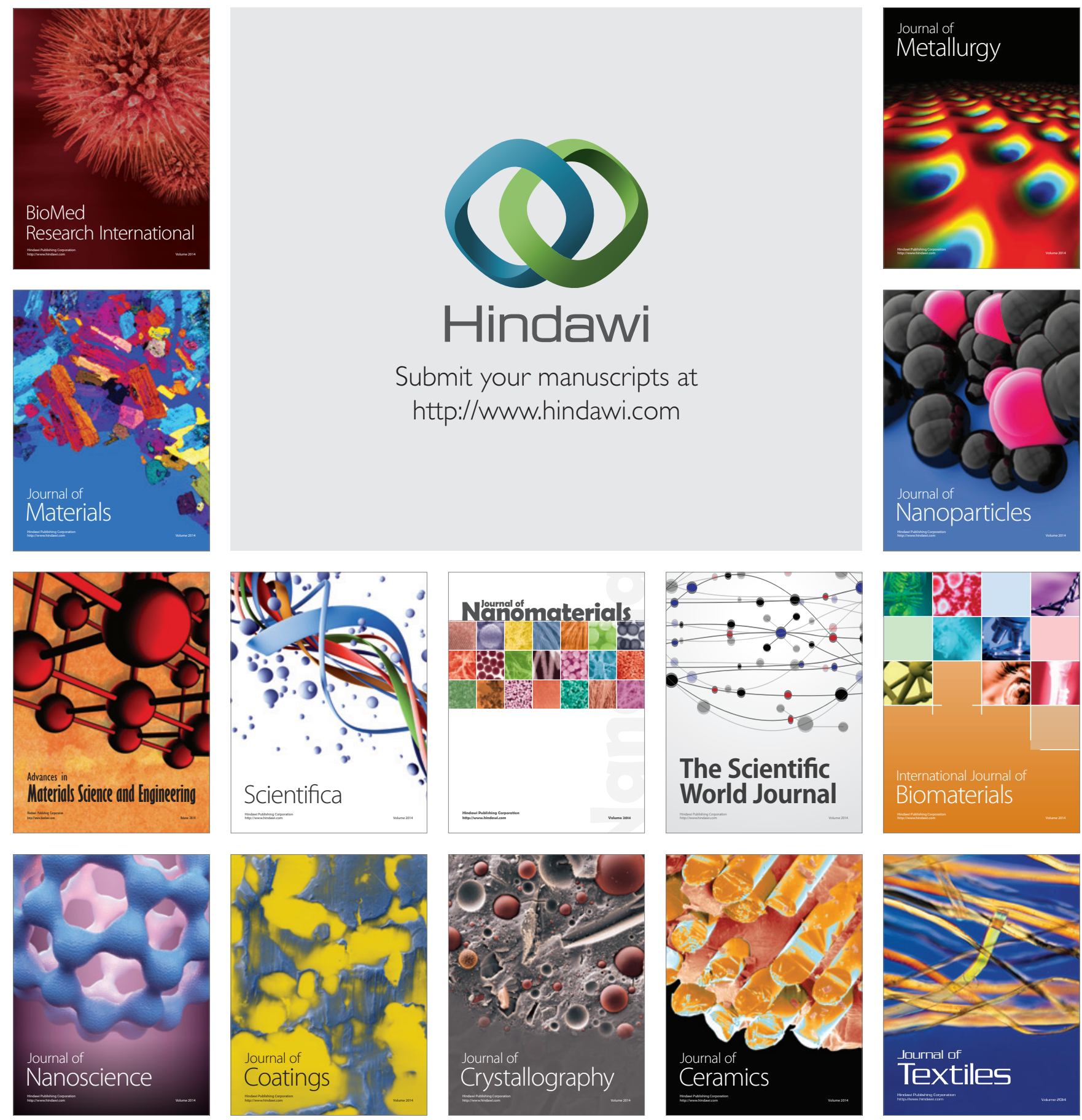